\section{Prévalences \\ du VIH en Afrique sub-saharienne}

\section{Historique d'une estimation}

Joseph Larmarange
> Tous les deux ans, le programme commun des Nations-Unies sur le VIH/SIDA (ONUSIDA) publie des estimations détaillées du nombre de personnes infectées par le VIH (virus de l'immunodéficience humaine). Selon la dernière édition de son rapport, publiée en juin 2008, 33 millions de personnes (adultes et enfants) dans le monde dont 22 en Afrique subsaharienne seraient infectées en 2007 . Cette estimation, largement reprise par les médias et les acteurs de la lutte contre le SIDA, a été révisée à maintes reprises ces dernières années. À titre d'exemple, le rapport 2002 estimait ce nombre à 28,5 millions en 2001 pour l'Afrique subsaharienne. Cette même estimation a été révisée à la baisse, dans la dernière édition du rapport, à 20,4 millions en 2001. Paradoxalement, le nombre de personnes infectées a augmenté entre 2001 et 2007 . Les révisions successives effectuées par l'ONUSIDA ont semé le doute sur la qualité des estimations produites. Petit retour sur l'évolution des sources et des techniques. <

Les estimations de I'ONUSIDA concernant le nombre de personnes infectées et la prévalence du VIH (proportion de personnes infectées dans la population), tant en Afrique subsaharienne que dans le monde entier, ont été révisées à plusieurs reprises depuis le début des années 2000 [1]. Le flottement des estimations est en grande partie dû à la difficulté de la mesure. Au fil du temps se sont mises en place différentes méthodes d'observation: initialement de populations ciblées puis de la population générale. Pour mieux comprendre la qualité des estimations actuelles et les raisons de ces révisions successives, nous nous proposons de retracer ici l'évolution des sources de données et des méthodes d'estimation utilisées pour mesurer les épidémies en Afrique subsaharienne (voir [2] pour une synthèse des spécificités des épidémies africaines).

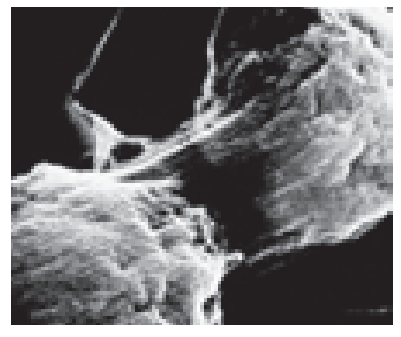

Centre Population et Développement (CEPED), UMR 196 Paris Descartes, INED, IRD, Université Paris Descartes, 45, rue des Saints-Pères, 75006 Paris, France. joseph.larmarange@ceped.org

\section{Les années 1980 : mise en place d'une surveillance sentinelle}

Les premiers cas de SIDA sont décrits au début des années 1980 aux États-unis et en Europe chez des patients homosexuels masculins. II faut néanmoins attendre le 21 novembre 1986 pour que les premiers chiffres concernant le nombre de cas de SIDA en Afrique soient publiés par I'OMS (organisation mondiale de la santé) [3].

Le développement, à partir de 1984, de tests de dépistage permet la mise en place d'enquêtes pour mesurer la prévalence du VIH. L'OMS, dans le cadre du Programme Global sur le SIDA, va formaliser à la même époque les principes d'une surveillance sentinelle. II s'agit de mettre en place des enquêtes de prévalence, auprès de populations choisies, afin de suivre les tendances des épidémies.

Les enquêtes nationales en population générale s'avèrent alors trop coûteuses pour être menées en routine [4] et souffrent de taux de participation relativement faibles. Or, en 1988, deux études menées respectivement aux ÉtatsUnis [5] et en Norvège [6], montrent que la prévalence est plus élevée parmi les personnes refusant de se faire tester. S'appuyant sur ces résultats, l'OMS préconise alors la méthode UAS (unlinked anonymous testing) qui consiste à tester des échantillons sanguins, prélevés pour d'autres raisons que la surveillance $d u \mathrm{VIH}$, après suppression de toute donnée nominative.

De fait, la surveillance sentinelle proposée par l'OMS va porter sur des sous-populations facilement accessibles: femmes enceintes, patients atteints d'IST (infections sexuellement transmissibles), donneurs de sang, travailleuses du sexe... Les femmes enceintes constituent une population facile à étudier puisqu'une prise de sang 
est réalisée dans le cadre du suivi prénatal. La surveillance sentinelle des femmes enceintes consiste donc à sélectionner certaines cliniques prénatales réparties sur le territoire national, puis à prélever un échantillon sanguin pour chaque femme lors de son premier entretien prénatal. Ce type d'enquête peut facilement être réalisé annuellement avec pour objectif de mesurer les tendances des épidémies pour permettre l'évaluation des activités et des stratégies de prévention. «/l doit être noté que les populations sentinelles ne nécessitent pas d'être "représentatives" » [7]. L'hypothèse sous-jacente repose sur le fait que si la collecte des données est uniforme au cours du temps, ainsi que les biais de sélection, alors les tendances observées refléteront les tendances réelles.

\section{Les années 1990 :}

\section{estimer la prévalence nationale du VIH pays par pays}

Au début des années 1990, alors que l'épidémie se généralise dans bon nombre de pays, l'observation des seules tendances ne suffit plus. II devient crucial de disposer d'une estimation du niveau de la prévalence du VIH. La majorité des modèles épidémiologiques développés jusqu'alors porte sur le nombre de cas de SIDA, à l'exception d'un modèle

\begin{tabular}{lccccccc}
\hline & & \multicolumn{2}{c}{ ENPG } & \multicolumn{5}{c}{ Estimation du rapport ONUSIDA } \\
\multicolumn{1}{c}{ Pays } & Enquête & Publication & $\%$ & 2002 & 2004 & 2006 & 2008 \\
\hline Burkina Faso & 2003 & 2004 & 1,8 & 6,5 & 4,2 & 2,0 & 1,6 \\
\hline Cameroun & 2004 & 2005 & 5,5 & 11,8 & 6,9 & 5,4 & 5,1 \\
\hline Côte d'lvoire & 2005 & 2006 & 4,7 & 9,7 & 7,0 & 7,1 & 3,9 \\
\hline Éthiopie & 2005 & 2006 & 1,4 & 6,4 & 4,4 & n. d. & 2,1 \\
\hline Ghana & 2003 & 2004 & 2,2 & 3,0 & 3,1 & 2,3 & 1,9 \\
\hline Guinée & 2005 & 2006 & 1,5 & n. d. & 3,2 & 1,5 & 1,6 \\
\hline Kenya & 2003 & 2004 & 6,7 & 15,0 & 6,7 & 6,1 & n. d. \\
\hline Lesotho & 2004 & 2005 & 23,5 & 31,0 & 28,9 & 23,2 & 23,2 \\
\hline Malawi & 2004 & 2005 & 11,8 & 15,0 & 14,2 & 14,1 & 11,9 \\
\hline Mali & 2001 & 2002 & 1,7 & 1,7 & 1,9 & 1,7 & 1,5 \\
\hline Ouganda & 2004 & 2006 & 6,4 & 5,0 & 4,1 & 6,7 & 5,4 \\
\hline Rwanda & 2005 & 2006 & 3,0 & 8,9 & 5,1 & 3,1 & 2,8 \\
\hline Sénégal & 2005 & 2006 & 0,7 & 0,5 & 0,8 & 0,9 & 1,0 \\
\hline Tanzanie & 2003 & 2005 & 7,0 & 7,8 & 8,8 & 6,5 & 6,2 \\
\hline Zambie & 2001 & 2003 & 15,6 & 21,5 & 16,5 & 17,0 & 15,2 \\
\hline
\end{tabular}

Tableau I. Prévalences du VIH (âge 15-49 ans, en \%) estimées à partir d'enquêtes nationales en population générale (ENPG) et selon plusieurs rapports ONUSIDA pour une sélection de pays africains (Source: adapté de [19], Figure 1.8 pages 52-53, et complété de [1]). Pour les enquêtes nationales en population générale ( $\varepsilon N P G)$, sont indiquées l'année de réalisation de l'enquête et l'année de publication des résultats. nd : non disponible dans le rapport ONUSIDA concerné. de l'OMS estimant les évolutions de la prévalence du VIH, qui sera mis en ligne fin 1991 sous le nom d'EpiModel [8]. II requiert a minima une estimation de la prévalence du VIH à une date donnée et une estimation de l'année de démarrage de l'épidémie. En 1992, dans l'ouvrage collectif AIDS in the World [9], sont publiées des estimations par grandes régions géographiques réalisées à l'aide d'EpiModel et affinées à partir de panels d'experts. En décembre 1995, les premières estimations pays par pays sont réalisées par l'OMS [10], toujours avec EpiModel et à partir des données de surveillance sentinelle obtenues dans les cliniques prénatales, les femmes enceintes étant considérées comme représentatives de la population générale (15-49 ans).

L'ONUSIDA est créé le $1^{\text {er }}$ décembre 1995. En juin 1998 sort son premier rapport mondial. Les estimations pays par pays fin 1994 sont alors actualisées fin 1997, toujours avec EpiModel, puis fin 1999 pour le rapport 2000. Cette même année, I'ONUSIDA décide de lancer une initiative pour une surveillance sentinelle de seconde génération [11]. Les zones rurales sont sous représentées et la qualité globale de la surveillance a diminué à la fin des années 1990 [12]. La surveillance de seconde génération préconise alors une augmentation du nombre de sites ruraux, une collecte des caractéristiques sociodémographiques des femmes testées et la mise en place d'enquêtes comportementales pour évaluer les progrès de la prévention [11].

En 1999, l'ONUSIDA créé un Groupe de Référence en Epidémiologie qui deviendra en 2002 le Groupe de Référence d'ONUSIDA sur les Estimations, la Modélisation et les Projections (http://www.epidem.org). II a pour objectif de faire évoluer les outils utilisés pour les estimations nationales. Il élabore un nouveau modèle reposant sur une approche épidémiologique. Ce modèle divise la population des individus de quinze ans et plus en trois groupes: les personnes sans risque d'être infectées, les personnes à risque et les personnes infectées. II peut être résumé en quatre paramètres: l'année de début de l'épidémie, la proportion initiale de la population à risque, l'intensité de l'infection et un paramètre comportemental qui affecte la répartition des nouveaux entrants entre personnes à risque et sans risque. Le modèle définit ainsi un type de courbes d'évolution temporelle de la prévalence du VIH. Un ajustement statistique est réalisé pour déterminer quelle combinaison de paramètres produit 
la courbe la plus proche des observations réalisées parmi les femmes enceintes. Ce modèle est implémenté dans un logiciel appelé EPP pour Epidemic Projection Package (rebaptisé Estimation and Projection Package en 2004), téléchargeable sur http://www.unaids.org. II sera utilisé pour la première fois pour les estimations présentées dans le rapport 2002.

Pour le rapport 2004, EPP est perfectionné. L'interface du logiciel est améliorée et dorénavant deux courbes différentes sont estimées: I'une pour le milieu urbain et l'autre pour le milieu rural, toujours à partir de l'observation des femmes enceintes. Une estimation nationale est ensuite réalisée en tenant compte de la répartition de la population par milieu de résidence [13]. Pour la première fois, le rapport fournit des estimations à deux dates, en l'occurrence à la fin de 2003 et à la fin de 2001, et des marges d'incertitudes autour des estimations [14]. L'impact démographique (décès, orphelins, etc.) est estimé à partir du module AIDS Impact Model du logiciel Spectrum qui se fonde sur les estimations réalisées avec EPP [15].

\section{Les années 2000 : le retour des enquêtes en population générale}

Au début des années 2000, il est envisagé d'avoir recours à des enquêtes nationales en population générale, à la suite de plusieurs débats éthiques et de l'adoption de procédures assurant la confidentialité des données. Il s'agit d'introduire un module de dépistage dans des enquêtes déjà existantes (notamment les enquêtes démographiques et de santé ou EDS conduites en routine tous les quatre à cinq ans). Une première enquête est ainsi conduite au Mali en 2001, suivie par plusieurs dizaines d'autres depuis.

Les résultats de ces enquêtes divergent parfois largement des estimations produites jusqu'alors (Tableau I). Dès 2003, trois chercheurs de I'OMS et de I'ONUSIDA publient un article dans le Lancet où ils précisent la nécessité de mieux comprendre les différences observées entre les chiffres résultant de la surveillance des femmes enceintes et des enquêtes nationales en population générale [16]. Ils discutent notamment la sous représentativité du milieu rural dans la surveillance sentinelle qui induirait une surestimation, et les taux de non participation relativement élevés des enquêtes nationales entraînant une sous-estimation. Le débat sur les taux de non participation s'est calmé depuis la publication de plusieurs travaux montrant que le biais s'avérait faible [17-21]. D'autres biais ont même été explorés, et il s'est avéré que l'erreur induite était inférieure à l'imprécision statistique des enquêtes [19]. À titre d'exemple, dans l'EDS menée en 2003 au Kenya, la correction de cinq sources de biais induit une prévalence ajustée comprise entre 6,55 et $7,16 \%$, tandis que la prévalence mesurée dans l'enquête est de $6,88 \%$ (intervalle de confiance à $95 \%$ : $6,27-7,54)$. Les enquêtes en population générale constituent donc de bons indicateurs du niveau de la prévalence du VIH au niveau national, à condition de ne pas leur conférer une précision supérieure à la leur. Concernant la surveillance sentinelle des femmes enceintes, leur « représentativité » a longtemps été justifiée par des comparaisons locales, dans le cadre d'observatoires de populations, entre prévalence mesurée en population générale et prévalence mesurée en clinique prénatale. Or, à

Figure 1. Comparaisons locales entre prévalence du VIH observée en clinique prénatale et prévalence du VIH en population générale (hommes et femmes) (Source: extrait de [19], Figure 3.20, page 186). 
une échelle locale, il apparaît que la prévalence observée dans les cliniques prénatales sous-estime la prévalence chez l'ensemble des femmes du fait d'une fécondité moindre des femmes séropositives [22]. Comme sur le continent africain les hommes sont, le plus souvent, moins infectés que les femmes, les deux biais se compensent en partie et la prévalence des femmes enceintes s'avère plus ou moins proche de celle de l'ensemble des adultes (hommes et femmes) (Figure 1), sous réserve que la population «captée » par la clinique prénatale soit représentative de la population locale, ce qui n'est pas forcément le cas dans les grands centres urbains où les femmes ont le choix parmi plusieurs centres de prise en charge.

\begin{tabular}{|c|c|c|c|c|c|}
\hline \multirow[b]{2}{*}{ Pays } & \multirow[b]{2}{*}{ Année } & \multicolumn{2}{|c|}{ Urbain } & \multicolumn{2}{|c|}{ Rural } \\
\hline & & ENPG & CPN & ENPG & CPN \\
\hline Bénin & 2006 & 1,7 & 1,9 & 0,9 & 1,2 \\
\hline Burkina Faso & 2003 & 3,6 & 3,9 & 1,3 & 1,6 \\
\hline Burundi & 2002 & 9,4 & 11,8 & 2,5 & 2,5 \\
\hline Cameroun & 2004 & 6,7 & 8,3 & 4,0 & 6,8 \\
\hline Côte d'Ivoire & 2005 & 5,4 & 6,7 & 4,1 & 4,6 \\
\hline Éthiopie & 2005 & 5,6 & 10,5 & 0,7 & 1,9 \\
\hline Ghana & 2003 & 2,3 & 3,5 & 2,0 & 3,3 \\
\hline Guinée & 2005 & 2,4 & 5,0 & 1,0 & 3,2 \\
\hline Kenya & 2003 & 10,0 & 11,0 & 5,6 & 8,6 \\
\hline Lesotho & 2004 & 29,1 & 32,3 & 21,9 & 24,3 \\
\hline Malawi & 2004 & 18,3 & 18,4 & 11,3 & 12,8 \\
\hline Mali & 2001 & 2,2 & 3,6 & 1,5 & 2,6 \\
\hline Niger & 2002 & 2,1 & 2,5 & 0,6 & 1,3 \\
\hline Ouganda & 2004 & 10,1 & 9,2 & 5,7 & 4,3 \\
\hline République Centrafricaine & 2006 & 8,3 & 13,4 & 4,7 & 15,4 \\
\hline Rwanda & 2004 & 7,3 & 5,9 & 2,2 & 2,7 \\
\hline Sénégal & 2005 & 0,7 & 1,5 & 0,7 & 1,4 \\
\hline Tanzanie & 2004 & 10,9 & 11,2 & 5,3 & 5,5 \\
\hline Tchad & 2005 & 7,0 & 4,9 & 2,3 & 2,8 \\
\hline Zambie & 2002 & 23,1 & 26,3 & 10,8 & 12,7 \\
\hline Zimbabwe & 2005 & 18,1 & 21,9 & 17,6 & 18,8 \\
\hline
\end{tabular}

Tableau II. Comparaison nationale des prévalences du VIH (âge 15-49 ans, en \%) estimées à partir d'enquêtes nationales en population générale (ENPG) et de la surveillance sentinelle en cliniques prénatales (CPN) pour une sélection de pays africains (Source : adapté de [29]). Les prévalences en cliniques prénatales ont été calculées à partir d'EPP, en utilisant la procédure d'ajustement de niveaux (level fit), sans calibrage sur les données des enquêtes nationales en population générale.
Au niveau national, en revanche, la situation varie très fortement d'un pays à l'autre (Tableau II) : la prévalence nationale estimée via les cliniques prénatales dépend essentiellement de la localisation des sites sentinelles retenus, l'épidémie n'étant pas répartie uniformément sur le territoire. Comme les sites sentinelles ont le plus souvent été choisis en milieu urbain et dans les zones suspectées «à risque », les estimations passées d'ONUSIDA ont le plus souvent surestimé la réalité (Tableau I). Plusieurs améliorations ont été apportées au logiciel EPP pour le rapport 2006 de l'ONUSIDA. Tout d'abord une procédure nommée level fit (ajustement de niveaux) permet de prendre en compte l'extension progressive des systèmes de surveillance sentinelle, notamment en milieu rural [23]. Mais surtout, il devient possible de calibrer les courbes obtenues, soit sur les résultats d'une enquête en population générale lorsque celle-ci est disponible, soit en réduisant de $20 \%$ les estimations obtenues en milieu rural. Si les enquêtes en population générale fournissent une estimation précise du niveau de l'épidémie une année donnée, il manque des données de tendances pour estimer les évolutions. La surveillance sentinelle des femmes enceintes, quant à elle, fournit des séries de données répétées à intervalles réguliers. L'approche d'ONUSIDA consiste donc à estimer le niveau de l'épidémie à partir des enquêtes en population générale et les tendances à partir de la surveillance des femmes enceintes.

\section{Et dans le futur?}

D'autres enquêtes nationales en population générale sont en cours et il est probable que l'on assiste encore à quelques réajustements dans le rapport 2010. Certains pays vont prochainement disposer des résultats de deux enquêtes successives. II faudra donc vérifier la validité de l'hypothèse selon laquelle la surveillance des femmes enceintes traduit correctement les tendances de l'épidémie. Or cette hypothèse n'est valide que sous réserve que le recrutement des cliniques prénatales demeure comparable au cours du temps.

Les dynamiques épidémiques changent. La généralisation de l'accès aux antirétroviraux a ainsi un effet majeur sur la baisse de la mortalité des personnes infectées [24]. L'effet sur l'incidence (proportion de nouvelles infections) demeure quant à lui incertain [25], une remontée des prévalences n'étant pas à exclure. Or, le modèle EPP ne sait pas encore prendre en compte ce type de phénomènes. D'ores et déjà, d'après le groupe de référence de I'ONUSIDA, EPP n'arrive pas à reproduire certaines tendances observées aujourd'hui dans des pays comme le Kenya, l'Ouganda ou l'Afrique du Sud [26], où la prévalence du VIH s'est stabilisée après un pic et un début de 
baisse. Le groupe de référence envisage donc de faire évoluer les algorithmes d'ajustement utilisés et de rendre le modèle plus flexible, en permettant entres autres à certains paramètres de varier dans le temps.

Au final, pour chaque rapport, I'ONUSIDA ré-estime l'ensemble de la courbe épidémique : les estimations publiées ne peuvent être comparées d'un rapport à l'autre. La publication cette année d'une estimation rétroactive à fin 2001 permet d'appréhender enfin les tendances dans chaque pays. Si l'estimation du nombre total de personnes infectées a varié de manière importante, les grands traits de l'épidémie, notamment le poids relatif de chaque continent, n'ont pas changé. Des données de meilleure qualité s'avèrent néanmoins nécessaires pour mieux planifier les programmes d'actions et notamment estimer précisément les besoins en traitements et leurs coûts. Reste que les dynamiques épidémiques sont encore mal connues. Certaines populations vulnérables ont été sous-estimées dans les épidémies généralisées, comme les homosexuels masculins en Afrique [27]. Des travaux récents suggèrent qu'une part importante des nouvelles infections serait le fait de personnes elles-mêmes nouvellement infectées, ce qui induirait un effet «domino» [28]. Autant de domaines à explorer dans les années à venir. $\diamond$

\section{SUMMARY}

\section{HIV prevalence in sub-Saharan Africa:}

\section{background of an estimation}

In 2008 UNAIDS global report on AIDS, the number of people living with HIV in sub-Saharan Africa was estimated at 22 millions in 2007 and 20.4 millions in 2001, while in the 2002 report, the same estimation for 2001 was 28.5 millions. Changes in UNAIDS reflects evolutions of data sources and methods used for the estimates. Sentinel surveillance of pregnant women attending antenatal clinics (ANC) was developed in 1980's with the WHO recommendation of unlinked anonymous testing approach. The objective was not to be representative but to monitor trends. In the 1990's, as ANC data were available, they were used by EpiModel, a model developed by WHO for HIV prevalence estimates from 1992 to 2000. In 2002, a new epidemiological model called EPP (Estimation and Projection Package) was developed by the UNAIDS Reference Group on Estimates, Modelling and Projections, which, in countries with a generalised epidemic, is still based on ANC data collected over time. Since 2001, many countries have conducted national population-based surveys (NPS). Their results have often diverged from estimates based on ANC data. This was explained by the underrepresentation of rural clinics in sentinel surveillance and relative small participation rate in NPS. Since, several studies have shown that the impact of several biases (participation rate, non-household members, serological window of tests...) in NPS remains relatively low. NPS constitute a good indicator of HIV prevalence level. If pregnant women can be locally representative of the general population, at the national scale, it depends of the localization of selected clinics. But ANC provides data over time, which is not the case for NPS. The current approach of UNAIDS consists in estimating HIV prevalence trends from ANC and the level of the epidemics from NPS. But the hypothesis that ANC data are representative of trends still needs to be verified when several NPS will be available for a same country. $\diamond$

\section{RÉFÉRENCES}

1. ONUSIDA. Rapport sur l'épidémie mondiale de sida 2008. Genève : ONUSIDA, août $2008: 362 \mathrm{p}$. http://www.unaids.org/fr/KnowledgeCentre/HIVData/GlobalReport/2008/2008_Global_report.asp

2. Anglaret X, Salamon R. Épidémie de Sida en Afrique subsaharienne. Med Sci (Paris) 2004 ; $20: 593-8$.

3. OMS. SIDA, données mondiales. Relevé Épidémiologique Hebdomadaire 1986; 61 : 361-3.

4. Chin J. Public health surveillance of AIDS and HIV infections. Bull WHO $1990 ; 68: 529-36$.

5. Hull HF, Bettinger CJ, Gallaher MM, et al. Comparison of HIV-antibody prevalence in patients consenting to and declining HIV-antibody testing in an STD clinic. JAMA $1998 ; 260: 935-8$.

6. Jenum P. Anti-HIV screening of pregnant women in south-eastern Norway. NIPH Annals 1998 ; $11: 53-8$.

7. Slutkin G, Chin J, Tarantola D, Mann J. Sentinel surveillance for HIV infection: a method to monitor trends in population groups. Stockholm: IVth International Conference on AIDS, juin 1998: $8 \mathrm{p}$. http://whqlibdoc.who.int/hq/1988/WHO_GPA_DIR_88.8.pdf

8. Chin J, Lwanga SK. Estimation and projection of adult AIDS cases: a simple epidemiological model. Bull WHO $1991 ; 69$ : 399-406.

9. Mann J, Tarantola D, Netter TW. AIDS in the world: the global AIDS policy coalition. Cambridge : Harvard University Press, $1992: 1038 \mathrm{p}$.

10. OMS. Estimations de travail provisoires de la prévalence du VIH chez les adultes, à la fin 1994, par pays. Relevé Épidémiologique Hebdomadaire $1995 ; 70$ : 355-7.

11. UNAIDS/WHO working group on global HIV/AIDS and STI surveillance. Guidelines for second generation HIV surveillance. Genève : UNAIDS/WHO, $2000: 48$ p. http://data.unaids.org/ Publications/IRC-pub01/jc370-2ndgeneration_en.pdf?preview=true.

12. Garcia-Calleja JM, Zaniewski $\varepsilon$, Ghys PD, et al. A global analysis of trends in the quality of HIV sero-surveillance. Sexually Transmitted Infections $2004 ; 80$ (suppl 1) : i25-30.

13. Ghys PD, Brown T, Grassly NC, et al. The UNAIDS Estimation and projection package: a software package to estimate and project national HIV epidemics. Sexually Transmitted Infections 2004 ; 80 (suppl 1) : i5-9.

14. Grassly NC, Morgan M, Walker N, et al. Uncertainty in estimates of HIV/AIDS: the estimation and application of plausibility bounds. Sexually Transmitted Infections $2004 ; 80$ (suppl 1) : i31-8.

15. Stover J. Projecting the demographic consequences of adult HIV prevalence trends: the spectrum projection package. Sexually Transmitted Infections 2004 ; 80 (suppl 1) : i14-8.

16. Boerma JT, Ghys PD, Walker N. Estimates of HIV-l prevalence from national population-based surveys as a new gold standard. Lancet $2003 ; 362: 1929-31$.

17. Mishra V, Barrere B, Hong R, Khan S. Evaluation of bias in HIV seroprevalence estimates from national household surveys. Sexually Transmitted Infections 2008 ; 84 (suppl 1) : i63-70.

18. Mishra V, Vaessen M, Boerma JT, et al. HIV testing in national population-based surveys: experience from the Demographic and Health Surveys. Bull WHO 2006; 84 : 537-45.

19. Larmarange J. Prévalences du VIH en Afrique : validité d'une mesure. Thèse de doctorat en démographie : Université Paris Descartes, 2007 : 582 p. http://tel.archives-ouvertes.fr/tel00320283/fr/.

20. Larmarange J, Vallo R, Yaro S, et al. Estimating effect of non response on HIV prevalence estimates with DHS data. Marseille : $8^{\text {th }}$ AIDS Impact Conference, juillet 2007. http://www.aidsimpact. com/2007/Programme/abstract/?id=633.

21. Marston M, Harriss K, Slaymaker $\varepsilon$. Non-response bias in estimates of HIV prevalence due to the mobility of absentees in national population-based surveys: a study of nine national surveys. Sexually Transmitted Infections $2008 ; 84$ (suppl 1) : i71-7.

22. Zaba B, Gregson S. Measuring the impact of HIV on fertility in Africa. AIDS 1998 ; 12 (suppl 1) : S41-50.

23. Brown T, Grassly NC, Garnett G, Stanecki K. Improving projections at the country level: the UNAIDS estimation and projection Package 2005. Sexually Transmitted Infections 2006 ; 82 (suppl 3) : iii34-40.

24. Jahn A, Floyd S, Crampin AC, et al. Population-level effect of HIV on adult mortality and early evidence of reversal after introduction of antiretroviral therapy in Malawi. Lancet 2008; $371: 1603-11$.

25. Larmarange J. Accès aux antirétroviraux en Afrique et baisse de la mortalité. Transcriptases 2008 ; $137: 2-4$.

26. UNAIDS Reference Group for Estimates Modelling and Projections. Improving the EPP and Spectrum estimation tools for the 2008-9 round of national estimates with specific attention to prevalence fits and their uncertainty, changes in the urban:rural population ratio, bias in 
HIV prevalence measured in national surveys, incidence estimates, orphanhood estimates, effects of ART; and a discussion on concurrent partnerships. Londres: UNAIDS, février 2008 : 20 p. http://www.epidem. org/publications/London2008.pdf.

27. Larmarange J. Homosexuels masculins : les oubliés de la prévention? Transcriptase/ANRS Information 2008; 138 (sous presse).

28. Brenner $B G$, Roger M, Routy JP, et al. High rates of forward transmission events after acute/early HIV-1 infection. J Infect Dis 2007 ; 195 : 951-9.
29. Gouws $\varepsilon$, Mishra V, Fowler TB. Comparison of adult HIV prevalence from national populationbased surveys and antenatal clinic surveillance in countries with generalised epidemics: implications for calibrating surveillance data. Sexually Transmitted Infections 2008; 84 (suppl 1) : i17-23.

TIRÉS À PART

J. Larmarange

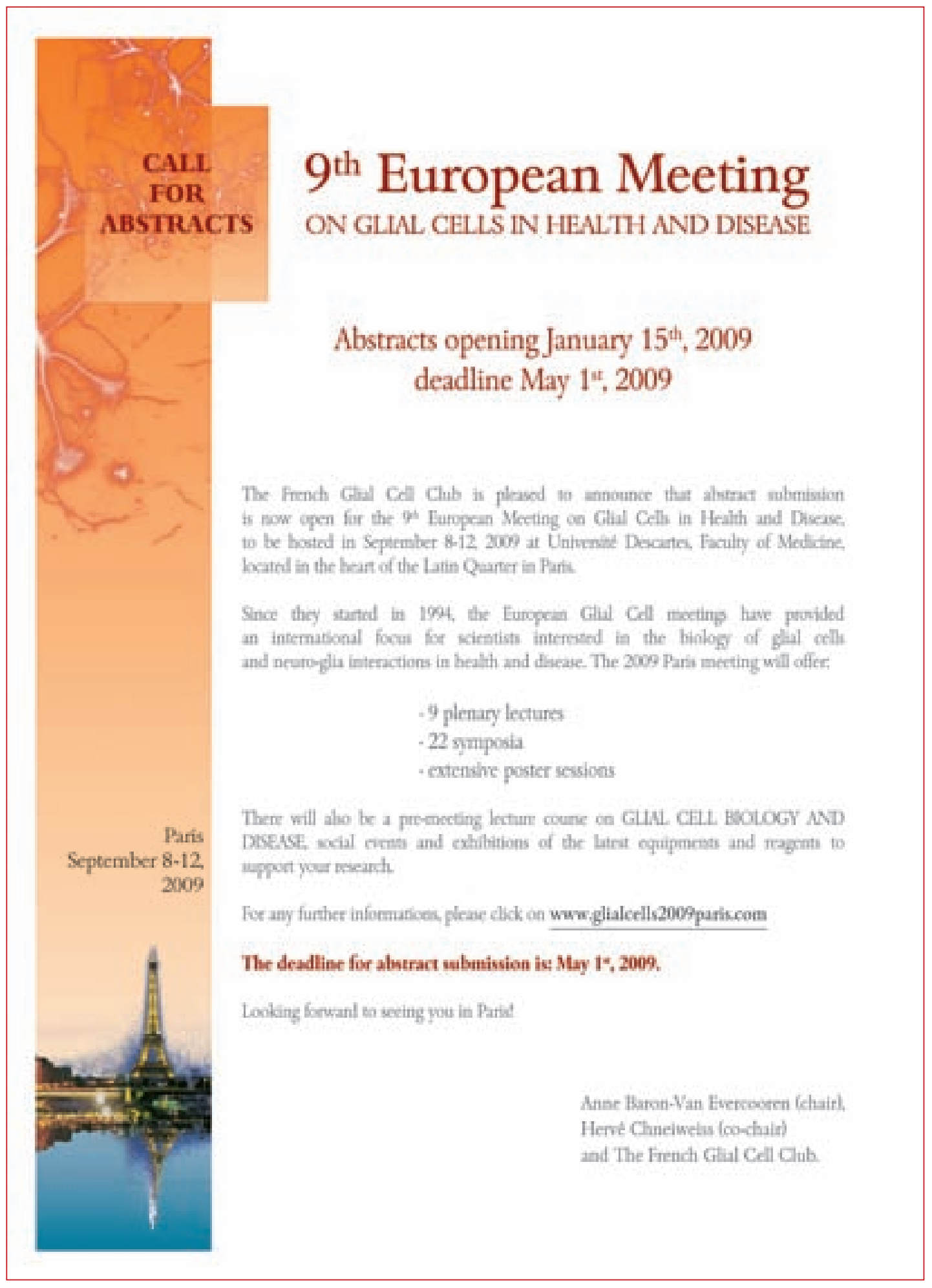

